\title{
Transformaciones en el sistema alimentario y cambios de dieta en España durante el siglo XX
}

\author{
Alicia LANGreo y LUIS Germán
}

\begin{abstract}
PALABRAS CLAVE: sistema alimentario, industria alimentaria, distribución alimentaria, cambio alimentario.
\end{abstract}

\section{CÓDIGOS JEL: F10, L66, N64, Q13.}

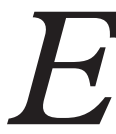

ste artículo reflexiona sobre cómo los cambios en el sistema alimentario espanol han sido determinantes en los cambios en la disponibilidad y en el consumo alimentarios del siglo $x x$. Se han diseñado varias etapas y se ha trabajado con algunos de los sectores en los que se han experimentado algunos de los principales cambios alimentarios. La primera etapa se refiere a las primeras décadas del siglo, antes de la Guerra Civil y la inmediata posguerra; la segunda abarca desde los primeros años cincuenta al inicio de la década de los setenta; y la tercera, desde entonces hasta el final del siglo.

A lo largo de los tres primeros cuartos del siglo se produjeron importantes innovaciones, tanto en el sector agrario como en el industrial, en contraste con una tradicional distribución minorista muy deficiente. En el cuarto apartado del artículo se analizan los importantes cambios que se han producido durante el último cuarto de siglo en el ámbito de la distribución alimentaria y su creciente control de las cadenas del sistema alimentario, vinculados a la rápida expansión y creciente concentración de la gran distribución minorista. Cambios que han condicionado los tradicionales hábitos de compra y de consumo, en un contexto de desplazamiento del gasto alimentario hacia la diversificación de productos de mayor calidad, más saludables, procesados y personalizados; así como el creciente peso del consumo alimentario fuera del marco doméstico. 


\section{Transformations in the food system and the role of industrial and food distribution changes in the Spanish diet during the twentieth century}

\section{KEYWORDS: food system, food industry, food distribution, dietary changes.}

\section{JEL CODES: F10, L66, N64, Q13.}

his article reflects on how changes in the Spanish food system have been in-
strumental in changing the availability and consumption of foods in the twen-
tieth century. We designed several stages in the study and examined several sectors that have experienced major changes. The first stage refers to the first decades of the twentieth century, prior to the Spanish Civil War, and immediately after the war; the second spans from the early fifties to the beginning of the seventies; and the third go from then until the end of the century.

Throughout the first three quarters of the century there were major innovations in agriculture and industry, in contrast to a very poor traditional retail distribution system. In the fourth sections of the article, major changes that occurred during the last quarter of the century in food distribution and growing control over the food chain are analyzed in relation to the rapid expansion and increasing concentration of large-scale retail distribution. These changes affected traditional buying habits and consumption, shifting food expenditures towards higher-quality, healthier, processed and personalized goods; as well as increasing food consumption outside the home.

Recepción: 2014-06-06 - Revisión: 2016-09-23 - Aceptación: 2016-12-01

Alicia Langreo es doctora ingeniera agrónoma, directora de la Sociedad de Estudios Saborá SL Estrategias Agroalimentarias. Dirección para correspondencia: c/ Hileras, 10, 28013, Madrid.C.e.: alicia_langreo@, sabora.es

Luis Germán Zubero [orcid.org/0000-0003-3977-7098] es catedrático de Historia e Instituciones Económicas en la Universidad de Zaragoza e investigador del Instituto Agroalimentario de Aragón (IA2). Dirección para correspondencia: Departamento de Estructura, Historia Económica y Economía Pública, Facultad de Economía y Empresa, Universidad de Zaragoza, GranVía 4, 50005 Zaragoza. C.e.lgerman@unizar.es 


\section{INTRODUCCION}

A lo largo del siglo xx la alimentación ha sufrido cambios drásticos muy rápidos. Para que éstos tuviesen lugar, han sido necesarias transformaciones radicales en la producción y comercialización de alimentos, es decir, en el sistema alimentario (Malassis, 1997; Tansey \& Worsley, 1995) ${ }^{1}$. Estas transformaciones han permitido poner a disposición de los consumidores nuevos alimentos y formatos (Nicolau \& Pujol, 2011).

Las variaciones en la oferta de alimentos, tanto en cantidad como en calidad o formato, están relacionadas con la innovación, con los cambios empresariales en las distintas fases del sistema, con la política agraria y alimentaria, así como con el desarrollo de los mercados, especialmente a partir de la segunda globalización. Las transformaciones del sistema alimentario son a la vez una respuesta de éste a los cambios sociales, demográficos y económicos que modifican la demanda de alimentos; pero, paralelamente, su oferta condiciona la demanda (Langreo, 2008).

En este artículo se intenta hacer una interpretación sobre las transformaciones habidas en el sistema alimentario español (en sus componentes sectoriales) ${ }^{2}$ durante el siglo $\mathrm{XX}$ (Sanz Cañada, 1997) y cómo éstas han sido determinantes en algunos cambios en la disponibilidad y en el consumo alimentarios. Se han diseñado varias etapas (definidas por el sucesivo protagonismo de las diversas fases del sistema alimentario) y se repasan los principales cambios que han experimentado los diversos sectores. La primera etapa (protagonizada por el sector agrario) se refiere a las iniciales décadas del siglo, antes de la Guerra Civil y la inmediata posguerra (1900-1950); la segunda (vinculada al protagonismo industrial) abarca desde los primeros años cincuenta hasta el inicio de la crisis de los años setenta; y la tercera (protagonizada por la gran distribución minorista) desde entonces hasta el final del siglo. El estudio de cada etapa incorpora, asimismo, las principales características del consumo alimentario y la influencia que dichas transformaciones sectoriales han tenido en algunos cambios en la dieta.

1. Malassis define el sistema alimentario como la organización de las sociedades para producir y para consumir los alimentos. Incluye el estudio del sector agrario, de las industrias alimentarias (IA), de la distribución (DA), de la restauración, de las operaciones de importación-exportación, de las industrias y servicios a los que está ligado; así como del consumo alimentario. Una propuesta de aplicación al caso español fue recogida en GERMÁn, HERNÁNDEZ y MORENo (2009).

2. Una reciente revisión de las transformaciones del sector agroalimentario español (1960-2010) desde la perspectiva energética, en INFANTE, Aguilera y GonZÁleZ DE Molina (2014). 


\section{EL PROTAGONISMO AGRARIO: DE LOS AVANCES DURANTE EL PRIMER TERCIO DEL SIGLO XX AL RETROCESO DURANTE LA GUERRA E INMEDIATA POSGUERRA}

A principios del siglo xx la economía española -y su sistema alimentario- seguía manteniendo un perfil abrumadoramente agrario y rural: en torno a las dos terceras partes del total de sus activos y de su población se centraban en dichos ámbitos. Desde el punto de vista del consumo casi las dos terceras partes del gasto familiar se destinaba al gasto alimentario (Maluquer de Motes, 2013).

Asimismo, España contaba todavía con un nivel de consumo alimentario inferior al de las poblaciones de los países de Europa occidental. Durante el primer tercio del siglo en España creció el consumo de kilocalorías per cápita. Una dieta que siguió estando basada en el consumo de productos agrícolas frescos, rica en hidratos de carbono (cereales y patatas) y los tradicionales productos agroalimentarios de primera transformación. Se iniciaron, además, unos ciertos cambios en su composición, vinculados al avance del consumo de carne y lácteos (Cussó \& Garrabou, 2007), pero también de otros de origen vegetal, como el aceite, las patatas y el azúcar. Cambios en la dieta estrechamente relacionados con el crecimiento y la diversificación de la oferta agroalimentaria y con el inicio de los cambios estructurales en la economía y sociedad españolas (González de Molina et al., 2014).

Durante este periodo, la difusión de una nueva base energética inorgánica en España posibilitó las innovaciones del cambio técnico agrario (abonos químicos, aumento del riego mediante obras hidráulicas, maquinaria metálica, rotaciones más intensivas) que promovieron el crecimiento y diversificación de la producción agraria, así como de los rendimientos y de la productividad (Gallego, 1993). Este crecimiento intensivo, además, pudo contribuir al crecimiento del resto de la economía, propiciando, por un lado, un decidido proceso de cambio estructural (en treinta años se redujo en algo más de veinte puntos el peso de los activos del sector agrario, en beneficio del peso de los sectores industrial y de servicios), así como el más moderado cambio en la estructura del poblamiento (la reducción del peso rural en España fue solo de algo menos de diez puntos). Ello supuso ya un avance decidido de la industrialización (sobre todo del medio urbano, pero también aunque en menor medida del medio rural) y un menor avance de la urbanización. Estos cambios tendieron a concentrarse en la mitad septentrional peninsular.

A principios del siglo $\mathrm{xx}$ la industria española estaba protagonizada en gran medida por las industrias de bienes de consumo (alimentación y textil). Ambos subsectores representaban en 1900 en torno a las dos terceras partes del total de la cuota industrial, reducida en 1929 al 45\% (alimentación, pasó de un 40\% al 26\%). 
Casi la mitad de la cuota del subsector alimentario de 1900 se centraba en los molidos $(18 \%)$, sobre todo de granos (harinas, $13 \%$ ) y de aceitunas (aceites, 5\%). Los destilados suponían casi un $15 \%$, frente a las conservas (3\%) y los compuestos (4\%). Una industria, en su mayor parte de primera transformación de productos agrarios, que desde finales del siglo XIX había llevado a cabo la implantación de innovaciones tecnológicas en sus procesos fabriles que mejoraron su eficiencia productiva y la conservación de los productos (Di Vittorio \& Barciela, 2003).

El inicio de la diversificación de la oferta alimentaria durante el primer tercio del siglo se apoyó en el incremento de la demanda (en gran parte urbana) hacia productos de mayor elasticidad-renta (productos cárnicos y lácteos). Para el abastecimiento urbano de estos productos, se produjeron mejoras en el transporte (muy mayoritariamente ferroviario) aplicando pioneras técnicas de frío para la conservación de productos frescos en los desplazamientos. Asimismo, se inició el acercamiento de la producción cárnica hacia las ciudades (instalación de ganaderías en las cercanías, desarrollo de mataderos municipales), que contaron con crecientes medidas reguladoras municipales sobre higiene y control alimentario.

El papel de algunas empresas agroalimentarias innovadoras en nuevas ramas fue esencial en su desarrollo. Con todo, los productos de primera transformación siguieron teniendo el protagonismo en este periodo, lo que posibilitó su preferente localización en el medio rural. Dentro de una generalizada estructura de pymes, de empresas familiares de tipo personalista, surgieron ya algunas nuevas sociedades anónimas, destacando por su mayor tamaño las empresas azucareras-alcoholeras con factorías asentadas en las proximidades de las zonas de cultivo remolachero, que durante el periodo de entreguerras estuvieron especialmente localizadas en el valle medio del Ebro (Germán, 2003). En esos años todavía fue muy escasa la presencia del capital multinacional en la industria alimentaria española, en contraste con su mayor presencia en el ámbito exportador alimentario.

El subsector alimentario español constituyó durante la primera mitad del siglo la base exportadora de la economía española: cítricos levantinos, aceites y vinos (Pinilla \& Ayuda, 2009). En dichas actividades exportadoras ya fue muy relevante el papel de las empresas comercializadoras extranjeras, tanto respecto de la comercialización de los cítricos como del aceite o del vino. En estas ramas productivas, cobró una creciente importancia la implantación de marcas y el embotellado.

La oferta alimentaria contaba todavía con un fuerte peso de los productos frescos y con productos manufacturados en general poco elaborados y con escaso envasado. En este 
escenario de limitados cambios productivos, la tradicional distribución alimentaria (repartida entre mayoristas y minoristas) no parece haber experimentado apenas transformaciones, si bien nuestro conocimiento del funcionamiento de este ámbito sigue siendo muy escaso (Nielfa, 1989; Gago, 2007; Sinde, 2014).

La guerra e inmediata posguerra civil española supusieron un claro retroceso, tanto en la producción como en los niveles de consumo, que solo recuperaron los índices de preguerra avanzada la década de los cincuenta. En la inmediata posguerra, durante los años cuarenta, se produjo una involución en el cambio estructural (medido en activos) de la economía española (García, 1957), su reagrarización, si bien prosiguió el proceso de urbanización.

\section{CUADRO 1}

Disponibilidades alimentarias en España, 1935-1972 (kg por habitante y año)

\begin{tabular}{lrrrr}
\hline & $\mathbf{1 9 3 5}$ & $\mathbf{1 9 5 0}$ & $\mathbf{1 9 6 0}$ & $\mathbf{1 9 7 2}$ \\
\hline Trigo & 156 & 90 & 105 & 70 \\
Leguminosas & 12 & 6 & 8 & 7 \\
Patata & 175 & 103 & 121 & 110 \\
Azúcar & 11 & 4 & 18 & 28 \\
Aceite & 11 & 11 & 15 & 16 \\
Carne & 33 & 13 & 20 & 45 \\
Huevos & 5 & 2 & 7 & 11 \\
\hline
\end{tabular}

*Las disponibilidades incluyen producciones y saldos del comercio exterior.

Fuente: Camilleri (1974), a partir de datos del Ministerio de Agricultura.

A pesar de este proceso de reagrarización, la producción agroalimentaria española -y su productividad-retrocedió. El sector agrario, afectado de un fuerte descenso de su capitalización (grandes carencias de abonos y maquinaria), se mostró incluso en algunos años incapaz de alimentar a su población, lo que llevó al Estado a la necesidad de importar grano para intentar garantizar la subsistencia de sus ciudadanos (Garrabou, 1997). La industria alimentaria también entró en retroceso productivo, afectada por problemas de abastecimiento de inputs (Catalán, 1994), pero también de falta de recursos energéticos y logísticos. Estas dificultades productivas se agravaron por una ineficiente política alimentaria, autárquica y fuertemente intervencionista (en 1937 se creaba el Servicio Nacional del Trigo; en 1941, la Comisaría de Abastecimientos y Transportes, CAT), que no impidió el desarrollo del mercado negro de productos alimenticios (Barciela, 1985). 


\section{DE LOS AÑOS CINCUENTA A LA CRISIS DE LOS SETENTA: EL PROTAGONISMO DE LA INDUSTRIA ALIMENTARIA}

Solo a partir de los años cincuenta se abrió un nuevo ciclo en el sistema alimentario español, crecientemente hegemonizado por la industria. El proceso de modernización agraria, vinculado a su progresiva capitalización e integración con el sector industrial, supuso la rápida crisis del segmento de agricultura tradicional (Naredo, 2004; Sumpsi, 1997) y la formación de modernos complejos agroindustriales (Juan i Fenollar, 1978). Las mejoras productivas de los principales complejos agroindustriales de este periodo (piensoganado-carne, leche), propiciaron ya el cambio del consumo alimentario hacia una orientación de mayor peso de las proteínas animales (Clar, 2008).

El crecimiento de dichos complejos no impidió la pérdida de peso del sistema alimentario dentro del conjunto de la economía española. Dentro de éste, fue perdiendo protagonismo el sector agrario en beneficio del sector industrial y de la distribución. Asimismo, desde el lado del consumo, fue perdiendo rápidamente peso el gasto alimentario dentro del total del gasto familiar.

\section{CUADRO 2}

Peso del gasto alimentario doméstico en España en el siglo xx

\begin{tabular}{lc}
\hline & (\% sobre el total de gastos) \\
\hline 1900 & 65,7 \\
1939 & 60,1 \\
1958 & 55,3 \\
$1973-1974$ & 38,0 \\
$1990-1991$ & 23,0 \\
2000 & 22,0 \\
\hline
\end{tabular}

Fuente: Maluquer de Motes (2013), a partir de las encuestas de presupuestos familiares (EPF) del Instituto Nacional de Estadística (INE).

A partir de los años cincuenta, España retomó el proceso de convergencia hacia los niveles de consumo de los países europeos occidentales, aunque conservando sus rasgos específicos. Una dieta que: a) aumentó su nivel nutricional/energético, aunque todavía protagonizada -si bien en retroceso- por cereales y patatas; b) recuperó el avance del consumo de carne (y pescado), lácteos, y frutas; y c) promovió un importante cambio de la estructura del consumo alimentario, ahora a favor de los alimentos de origen animal (Garrabou \& Cussó, 2009).

Estos cambios en el consumo fueron facilitados por la consolidación de una industria alimentaria, reforzada en este periodo con la llegada de capital extranjero desde los 
años sesenta, especialmente concentrada en sus ramas más expansivas ${ }^{3}$ (Peinado, 1985). Un consumo alimentario que mostraba, asimismo, el creciente, aunque todavía limitado, avance de nuevos productos transformados y procesados por la industria.

\section{CUADRO 3}

Estructura del gasto alimentario doméstico en España, 1958-1998(\%) ${ }^{\star}$

\begin{tabular}{lrrrr}
\hline & $\mathbf{1 9 5 8}$ & $\mathbf{1 9 7 5}$ & $\mathbf{1 9 8 7}$ & $\mathbf{1 9 9 8}$ \\
\hline Pan, pastas, cereales & 18,5 & 7,9 & 7,5 & 8,6 \\
Carnes & 17,6 & 29,6 & 27,9 & 25,4 \\
Patatas, hortalizas & 13,1 & 9,9 & 8,4 & 10,3 \\
Leche, queso, mantequilla & 8,7 & 10,4 & 13,0 & 12,6 \\
Aceite y grasas & 8,5 & 6,7 & 4,8 & 3,0 \\
Pescados & 8,3 & 8,8 & 10,9 & 12,6 \\
Huevos & 6,8 & 4,1 & 2,9 & 1,6 \\
Frutas y zumos & 5,3 & 7,1 & 9,8 & 8,9 \\
Vino, cerveza, licores & 4,4 & 5,7 & 3,6 & 4,5 \\
Azúcar y dulces & 4,2 & 3,7 & 5,6 & 5,5 \\
Café, malta y otros & 2,1 & 2,3 & 1,6 & 1,4 \\
Bebidas no alcohólicas & 0,3 & 1,4 & 1,5 & 2,6 \\
Otros & 2,2 & 2,5 & 5,1 & 3,2 \\
\hline
\end{tabular}

*En el original, algunas columnas no suman 100 .

Fuente: Contreras (2002), a partir de las EPF del INE, y de datos del Ministerio de Agricultura y Pesca, Alimentación y Medio Ambiente.

\subsection{Formación y expansión de modernos complejos agroindustriales}

En los años cincuenta se inició el incremento del consumo de carne, que respondía a una mejora paulatina de la renta, consolidada durante los años del desarrollismo, y a las mejoras productivas de la cadena ganadero-cárnica, protagonizada por multinacionales, que permitieron un descenso de los precios. El creciente consumo de carne, que alcanzaba en 1975 en torno al 30\% del gasto alimentario doméstico, pasó a ser su principal componente (Cuadro 3). En este marco hubo importantes cambios en la política económica de esos años que introdujeron modificaciones fundamentales en el sistema alimentario, especialmente el incremento del consumo de carne, sobre todo de pollo y cerdo (Soria, Delgado \& Rodríguez Zúñiga, 1976). Fue especialmente relevante el diseño de una nueva política ganadera, iniciada en dicha década y consolidada en los años sesenta, que impulsó

3. Las industrias cárnicas, de sacrificio de ganado y piensos compuestos, lácteas, derivados del aceite, frente a la pérdida de peso de ramas con productos de menor elasticidad-renta. 
la creación de nuevos regadíos, la difusión de variedades más productivas de cereales y ganado, la utilización intensiva de nuevos tipos de maquinaria y energía inanimada (Rodríguez Zúñiga, Ruiz Huertas \& Soria, 1980), y que se basaba en los mecanismos de coordinación vertical en la cadena de producción (Langreo, 1978). Esta política puso las bases del desarrollo del complejo producción de piensos-ganadería intensiva-producción de carne, que implicó cambios profundos en la política de cereales y de oleaginosas: se acabó la preferencia por el trigo y se potenció la producción de cebada y maíz. La producción total de granos entre 1940 y 1970 se duplicó, y se introdujo la soja y el girasol en las rotaciones de cultivos (Clar, 2005). Asimismo, se consolidó una corriente importadora de maíz y de soja que fue fundamental para la creciente producción de carne a un precio asequible (Viladomiú, 1985).

El desarrollo ganadero intensivo se basó en la introducción de razas especializadas en la producción de carne, con el consiguiente arrinconamiento de las razas autóctonas, en la incorporación de criterios científicos en la alimentación ganadera y en la expansión de nuevas empresas de piensos que incorporaron la producción ganadera. Como resultado, entre 1950 y 1970 la producción de carne se multiplicó por casi cinco y el mapa ganadero español cambió de forma substancial: la ganadería intensiva se consolidó en Cataluña y las regiones vecinas, y en menor medida, en el entorno de Madrid, a la vez que las actividades de cría y cebo se separaron y dieron lugar a nuevas líneas de especialización. Las empresas de piensos jugaron un papel estratégico en la promoción de las nuevas actividades ganaderas entre los empresarios agrarios, con los que establecieron complejas relaciones de coordinación vertical. Este mecanismo permitió que el conjunto del sector tuviese un ritmo muy alto de incorporación de tecnología y un desarrollo económico y comercial muy importante, especialmente en los sectores avícola y porcino (Langreo, 1990; Rodríguez Zúñiga \& Langreo, 1992).

Paralelamente, se concentró el sector de los piensos, donde entró capital americano y francés, y se crearon grandes mataderos frigoríficos privados (principal componente de una nueva red de frío industrial propiciada por dicha iniciativa privada), de forma que la matanza de pollo y cerdo dejó de hacerse en los mataderos municipales. Asimismo, creció la industria de transformación de carne y se hicieron nuevas gamas que muy deprisa se integraron en la dieta de los españoles.

Entre los sectores con fuerte desarrollo en los años 1950 y 1960, destaca el sector lácteo, definitivamente potenciado por el Plan de Centrales Lecheras, que a fuerza de proteger la leche pasteurizada y su distribución en exclusiva en cada ciudad, terminó empujando a las empresas de mayor dimensión situadas en la cornisa cantábrica a la producción de leche estéril (que más tarde se adecuó mejor a las necesidades de la gran 
distribución), y acabó volcando el creciente consumo en ésta, especialmente cuando en los años setenta el método UHT solucionó los problemas organolépticos. El desarrollo del sector lácteo también incorporó un cambio en la base genética (generalización de la raza frisona, especializada en la producción láctea), pero no alteró la alimentación del ganado (Langreo, 1995; Collantes, 2014a).

En los años sesenta también se inició la producción intensiva de hortalizas extratempranas destinadas a la exportación en el sudeste peninsular y en Canarias y, posteriormente, la de algunas frutas de temporada y de fresas. Su desarrollo se basó en elevadas inversiones, alta incorporación de tecnologías, nuevas variedades, aprovechamiento de las condiciones agroclimáticas caracterizadas por unos recursos hídricos muy escasos y gran cantidad de sol, y, sobre todo, en el auge de un nuevo tipo de empresa muy capitalizada, capaz de integrar la producción agraria y la comercialización final. Como resultado, la exportación hortofrutícola creció muy deprisa y se consolidó como uno de los grandes recursos del sistema alimentario español. Sin embargo, las producciones intensivas de invierno tardaban en venderse en los mercados españoles, de forma que el cambio en la alimentación que supuso la disponibilidad de hortalizas frescas fuera de temporada no se produjo hasta finales de los setenta y principio de los ochenta. Esta prioridad a la exportación sobre el consumo nacional se encuentra en más productos y condicionó el consumo interno. Por ejemplo, el aceite de oliva fue uno de los productos cuyas exportaciones se primaron por motivos estratégicos (obtención rápida de divisas), mientras, en consonancia con la política ganadera, se fomentaba el consumo de aceite de soja y de girasol, cuya torta se destinaba a aportar la parte proteínica de los piensos (Tió, 1982).

El vino y el aceite continuaron siendo sectores muy importantes y extendidos en el territorio español, con un papel activo en la exportación agroalimentaria. El hecho más relevante del periodo fue la creación, con el patrocinio explícito del Estado, de una amplia red de cooperativas locales encargadas de la primera transformación, que dejaban el embotellado y la comercialización en manos de las grandes empresas con marcas propias. La producción masiva de graneles consolidó una baja calidad en una parte importante de la producción. El consumo español de vinos se dividía entre un escaso segmento de alta capacidad adquisitiva que consumía vinos embotellados de marca, especialmente Rioja, a los que podían sumarse los vinos espumosos y los especiales (sobre todo los de Jerez, orientados a la exportación), y el resto de la población, que consumía vinos comprados a granel (pellejos). A partir de los años cincuenta una serie de empresas (Savin, Casa) empezaron a embotellar en envases de litro, sustituyendo poco a poco los graneles (Fernández, 2012). 
El desarrollo experimentado por los sectores agrarios y agroindustrial en los años cincuenta y sesenta y la evolución de la sociedad dejaron obsoleta la política agraria diseñada en la posguerra y favorecieron diversas iniciativas institucionales encaminadas a favorecer la modernización de las diferentes fases del sistema alimentario ${ }^{4}$.

\subsection{Los orígenes de la gran distribución minorista: el autoservicio y las primeras cadenas}

Este periodo de creciente integración agroindustrial y de diversificación de la oferta alimentaria aumentó el peso de los productos no perecederos envasados, frente al anterior protagonismo de la venta a granel. Este proceso facilitó cambios en la distribución minorista alimentaria española, protagonizados desde los años sesenta por la difusión de la técnica de venta en autoservicio en la distribución de productos no perecederos: los pioneros supermercados y las primeras cadenas voluntarias ${ }^{5}$.

Las primeras formas de la gran distribución surgieron en España en la segunda mitad del siglo $\mathrm{xx}$, y muy pronto experimentaron un auge importante y empezaron a condicionar el resto de la cadena de producción de alimentos. El pronto éxito de las nuevas formas de la distribución moderna radicaba en que ofrecían soluciones más eficientes y que se adaptaban mejor a la nueva situación económica y social. En aquellos años la producción agraria e industrial estaba en condiciones de ofertar mayores cantidades a pre-

4. En el ámbito agrario se produjo la revisión en profundidad de la política de precios con la creación del FORPPA (Fondo de Ordenación y Regulación de los Precios de los Productos Agrarios), la modificación del SENPA (Servicio Nacional de Productos Agrarios, heredero del Servicio Nacional del Trigo), la aprobación del Estatuto del Vino, de la ley de APA (Asociaciones de Productores Agrarios) y del régimen especial agrario de la Seguridad Social. La nueva industria alimentaria se benefició de diversas iniciativas políticas, muchas de ellas en el marco de los planes de desarrollo. El Plan de la Red Frigorífica Nacional fue aprobado por el Gobierno en 1957 y un posterior Plan de Red de Transportes Frigoríficos en 1965. Por otro lado, en el ámbito de la política de comercio interior, la disolución de la CAT, la creación de la red de MERCA (la empresa pública MERCASA se constituyó en 1966) pusieron los cimientos para una mejora sustancial del comercio mayorista, sobre todo fresco, lo que repercutió en la mejora del suministro y de la calidad, permitiendo, por ejemplo, la distribución de más frutas y hortalizas en todas las regiones españolas (Reyero, 1991). A finales de 1970 se constituyó la Empresa Nacional de Mercados en Origen S.A. (MERCORSA) y en 1973 comenzó su actividad el Instituto para la Reforma de las Estructuras Comerciales (IRESCO) (CASARES \& REBOLLO, 1991).

5. Los modelos de autoservicio, con los que se revolucionó la distribución, se habían iniciado en Estados Unidos en vísperas de la Primera Guerra Mundial y se difundieron en este país durante el periodo de entreguerras. En la inmediata segunda posguerra se produjo un rápido proceso de americanización del comercio alimentario en Europa occidental (SCHRÖTER, 2008; SHAW, LOUISE \& ANDREW, 2004; LESCENT-GILES, 2005). 
cios más bajos; sin embargo, los sistemas tradicionales de distribución minoristas se mostraban lentos, largos y caros, a la vez que manejaban la mercancía con serias deficiencias. Las nuevas formas de distribución pronto empezaron a mostrar más eficacia, y encajaron mejor con el crecimiento y modernización de la producción de alimentos (Maixé, 2009; Castro \& Maixé, 2015).

La transformación del comercio minorista se vinculó generalmente a la existencia de un mayorista que a la vez les servía los productos y les asesoraba en la nueva forma de vender. En España, más tardíamente, se produjo, tanto la transformación de comercios ya existentes como la instalación de otros nuevos ya como autoservicio. Cabe mencionar que estas fórmulas, al igual que pasó con la modernización de la agricultura y de la industria, contaron con el apoyo de los gobiernos, cámaras de comercio y otras entidades. La evolución de todo el complejo que iba surgiendo fue muy flexible y dio lugar a diferentes fórmulas que básicamente se concretaron en torno a los modelos asociativos y a los sucursalistas, y en todos ellos los antiguos mayoristas tuvieron mucha importancia. Así, algunos mayoristas se asociaron para crear grandes centrales de compras y se insertaron en el ámbito minorista creando sus empresas con tiendas propias y franquicias.

Desde un primer momento, la entrada de las formas de venta en lineal mediante autoservicio obligaron a cambios en la industria y el comercio; los suministradores se vieron obligados a envasar el producto, alcanzar una cierta homogeneización y desarrollar las marcas. Esto sucedió, por ejemplo, con las legumbres, el arroz, los huevos, el azúcar; asimismo, las conservas y en general la alimentación seca envasada y marquista se movían muy bien en estos formatos de venta. Es decir, se favoreció un mayor desarrollo industrial, la identificación de los productos y la incorporación de una nueva actividad, el envasado, a las tareas de los comerciantes mayoristas e industriales, al igual que el crecimiento de la industria y de los suministradores comerciales, que también se vieron obligados a mejorar su logística.

En todo este proceso fue fundamental el hecho de que las empresas, asociadas en cadenas y centrales de compras, compraran grandes cantidades para suministrar a sus tiendas propias o asociadas, lo que relegaba al comercio mayorista que no participaba en el proceso, y les permitía llegar a acuerdos a medio plazo entre ellas y los industriales en cuanto a envasado, presentaciones, formas de entrega y pago. Los ámbitos geográficos de actuación de estas empresas en términos generales eran limitados, salvo algunas excepciones, y en general los acuerdos con suministradores abarcaban espacios concretos que fueron aumentando. Estos fueron los años de auge de la cadena Spar o del nacimiento de empresas regionales de distribución como Vegalsa (Grandío \& Maixé, 2008), Sabeco y Digsa (Germán, 2017) o Caprabo. 
El desarrollo de las nuevas formas de distribución tuvo un componente nacional muy fuerte: las empresas de distribución mantuvieron su actividad durante mucho tiempo en su país de origen y, como mucho, se expandieron a los vecinos dentro de Europa; en este punto las diferentes normativas legales que regían el comercio colaboraron en la limitación de la expansión. Si en todos los países la venta en lineal fue una de las señas de identidad de las nuevas formas comerciales, los tipos y dimensión de los comercios fueron muy diferentes.

Así, en España, hasta la llegada de los grandes hipermercados franceses en los años setenta y su auge en los noventa, la moderna distribución en lineal estaba protagonizada por empresas españolas con sucursales en territorios regionales limitados o bien por cadenas asociativas siguiendo el modelo alemán (Spar). En esta primera época se limitaban a ofrecer productos no perecederos, de larga duración, y sirvieron para potenciar el desarrollo del envasado y de las marcas. Sin embargo, su incidencia en el consumo todavía no fue excesivamente relevante.

En contraste, como se ha señalado antes, las mejoras productivas de los principales complejos agroindustriales de este periodo (pienso-ganado-carne, leche) orientaron el cambio del consumo alimentario hacia un mayor peso de las proteínas animales en la dieta.

\section{LOS CAMBIOS EN LA ALIMENTACION DESDE LA CRISIS DE LOS SETENTA: EL PROTAGONISMO DE LA GRAN DISTRIBUCION}

En el último cuarto de siglo, el consumo cuantitativo alimentario tocó techo en España. Se mantuvo ya una cierta estabilidad en su estructura (Cuadro 3) y fueron ganando rápidamente peso los alimentos procesados, alimentos sometidos a diversos procesos de transformación, los alimentos-servicio ${ }^{6}$. En las décadas finales del siglo se produjo una gran diversificación y segmentación del consumo, que multiplicó la oferta de dichos alimentos procesados, en el contexto de un nuevo modelo de consumo alimentario cuantitativamente estabilizado y crecientemente diversificado (Collantes, 2016).

Comenzó una preocupación creciente por los efectos de la alimentación en la salud, que convive con una gran incidencia de todo tipo de patologías alimentarias (obesidad,

6. El modelo alimentario basado en el alimento-servicio presenta los siguientes rasgos: elevado nivel de transformación, durabilidad, alta incidencia de la diferenciación y de la marca, incorporación de altos niveles de normalización y de información, utilidades procedentes del envasado, adaptación a las economías de tiempo, importancia de los criterios nutricionales y de salud. 
bulimia, anorexia). Se generaron muchos segmentos dentro del colectivo de consumidores: consumidores de productos ecológicos, vegetarianos en sus diversas acepciones, consumidores de lo local, colectivos que conceden mucha importancia a la calidad diferencial. En este sentido, se promovió el desarrollo de una amplia variedad de alimentos funcionales.

\section{CUADRO 4}

Estructura del gasto alimentario en España por tipos de productos, 1964-1991(\%)

\begin{tabular}{lrrr}
\hline & $\mathbf{1 9 6 4}$ & $\mathbf{1 9 7 3}$ & $\mathbf{1 9 9 1}$ \\
\hline Productos frescos & 34,0 & 33,4 & 22,3 \\
Transformados tradicionales & 24,8 & 15,9 & 12,0 \\
Transformados no tradicionales & 25,9 & 29,4 & 23,7 \\
Alimentos procesados & 15,3 & 21,3 & 42,0 \\
Total & $\mathbf{1 0 0 , 0}$ & $\mathbf{1 0 0 , 0}$ & $\mathbf{1 0 0 , 0}$ \\
\hline
\end{tabular}

Fuente: Abad y Naredo (1997) a partir de las EPF del INE.

Asimismo, ganó peso relativo el gasto alimentario fuera del hogar: pasó de representar en 1974 el 14,8\% del gasto total alimentario al 27,4\% en 1990 (Rama, 1997). A todo esto hay que sumar una cuestión fundamental: el hecho de que la percepción de la alimentación para una gran parte de las nuevas generaciones se acerca a la de otros consumos, con enorme influencia de la moda, la publicidad, la imagen, algo que a lo largo de la historia había tenido mucha menor influencia (Díaz Méndez \& González, 2008).

Desde el sistema alimentario, las importantes transformaciones habidas en la industria agroalimentaria y sobre todo en el ámbito de la distribución han influido en algunos cambios del consumo alimentario. Transformaciones sectoriales vinculadas al creciente protagonismo de la gran distribución minorista y su óptima utilización de las nuevas tecnologías de la información, y que -como analizamos más adelante en el epígrafe 4.2-con la puesta en marcha del Mercado Único se aceleraron en los años noventa. Por ello, presentamos sucesivamente las transformaciones habidas hasta finales de los años ochenta y durante la última década del siglo.

\subsection{Las transformaciones de la distribución y la industria alimentaria en los años ochenta}

El nuevo y creciente protagonismo de la gran distribución minorista obliga a exponer en primer lugar las causas de su auge. 
El nacimiento de la gran distribución fue una forma de equilibrar la cadena de producción de alimentos dentro del sistema alimentario. A lo largo del siglo xx éste había alcanzado un notable desarrollo y tanto el sector agrario como la industria, e incluso en menor medida las redes comerciales mayoristas, habían dado saltos enormes hacia la mejora de su eficiencia, pero se veían obligados a convivir con una distribución minorista fragmentada y deficiente, poco eficaz, que no se adaptaba a los fuertes cambios habidos en la sociedad. Ésa fue la clave de su rápido éxito. Más tarde, la concentración de sus decisiones de suministro y su excelente conocimiento del comportamiento del consumo (gracias a la rápida difusión del escaneado con lectores ópticos desde los primeros años ochenta), junto a la gran dimensión alcanzada, terminaron haciendo de la gran distribución la fase dominante en el sistema alimentario, lo que ha determinado en buena medida qué se produce y cómo, ha establecido protocolos a las etapas anteriores y ha obligado a todo el sistema alimentario a seguir su ritmo.

Desde entonces, la gran distribución ha protagonizado uno de los cambios fundamentales del sistema alimentario mundial, que ha coincidido con un salto adelante en la globalización del conjunto de la producción y distribución de alimentos y con fuertes tendencias a la homogeneización de determinados tipos de consumos y la extensión de marcas a escala mundial, un enorme incremento de los flujos internacionales de alimentos, tanto de materias primas como de alimentos frescos, elaborados y semielabo$\operatorname{rados}^{7}$.

Estos cambios a los que está vinculada directamente la gran distribución coinciden en el tiempo con otros de similar magnitud, que han afectado profundamente al sistema alimentario mundial: a) el ritmo de la innovación (en procesos, productos e ingredientes) y su rápida extensión, junto a la continuación de la revolución verde, en un proceso que exige enormes inversiones $y$, por lo tanto, capitales; b) los cambios en las políticas agrarias y alimentarias, con un avance hacia la limitación de las intervenciones y mecanismos de protección; c) el aumento de la dimensión de las empresas líderes del sistema alimentario mundial; d) el aumento de la incidencia de las estrategias de los grandes grupos financieros inversores; y e) los cambios en la alimentación de los países emergentes (Langreo, 2008; McMichael, 1995; Goodman \& Watts, 1997; Lang \& Heasman, 2004).

Las tendencias globalizadoras generalizadas han dejado huecos para el mantenimiento de producciones locales, que tienen que adaptarse continuamente a cada entorno y que se mantienen sostenidas por poblaciones locales que aman su gastronomía.

7. Sobre las causas del crecimiento del comercio mundial agroalimentario, SERRANO y PINILLA (2010). 
En el sistema alimentario español del periodo analizado en este apartado jugaron ya un papel fundamental las empresas de la gran distribución minorista. Con todo, todavía en 1987, primer año del Panel de consumo alimentario del Ministerio de Agricultura, Pesca y Alimentación, la tienda tradicional todavía contaba con una cuota mayoritaria en valor en toda la alimentación comprada en los hogares (Cuadro 6): el 53\% frente al 30\% de supermercados y el 3,5\% de los hipermercados (Martín Cerdeño, 2008). Era muy alto (por encima del 60\%) el peso del comercio tradicional en la alimentación considerada fresca (Cuadro 7). En los productos de alimentación seca, el peso del comercio tradicional era minoritario (algo más del 30\%), frente a la dominante posición de la moderna distribución (hay que tener en cuenta que entonces todavía tenían cierta relevancia algunos canales paralelos).

Dentro de la gran distribución minorista, en España el peso de los hipermercados hasta casi finales de los años ochenta siguió siendo pequeño, frente al protagonismo del formato supermercados (Cuadro 6): el 3,5\% frente al 30\% de la cuota de mercado. El mensaje básico del hipermercado era compre una sola vez por semana y compre barato y se adaptaba muy bien a los productos marquistas de larga duración, pero no parecía adecuado para los productos frescos, que más tarde han mostrado su importancia estratégica para el negocio de la distribución. Desde muy pronto los supermercados fueron mejorando sus cuotas de mercado y lograron situarse en los productos frescos, que nunca funcionaron bien en los hipermercados. Cabe mencionar que los supermercados han mantenido la ventaja de la proximidad de las tiendas de barrio y, con ella, la mayor frecuencia de visitas; por el contrario, sus costes logísticos han sido significativamente mayores y su gestión de suministro más complicada (Langreo, 2011).

Efectivamente, hasta principios de los años noventa el gran negocio de la distribución eran los productos de larga duración. Ellos fueron el objeto de las grandes batallas por ganar clientes, consistentes en una reducción de los precios (PVP) bajando los márgenes y presionando a los suministradores, bien en estrategias globales o en operaciones de ofertas puntuales. En España uno de los mejores ejemplos fue la leche líquida para el consumo, la leche esterilizada UHT, en la que también se desarrollaron muy pronto las marcas propias de la distribución (Langreo, 1995; Collantes, 2014b, 2015). Asimismo, el pan, un producto considerado fresco y caracterizado por su peso en la pequeña distribución minorista, la inexistencia de marcas y la presencia de un número muy alto de tahonas, fue objeto de importantes ofertas por parte de las grandes empresas de la distribución, aunque ésta tardó mucho en alcanzar una cuota de mercado significativa (Langreo, 2001). En ambos productos los efectos sobre los precios de venta al público fueron mayores a los correspondientes a la cuota que manejaban por entonces los hipermercados, poniendo de manifiesto la gran capacidad de arrastre de estos modelos comerciales. 
Ambas ramas industriales se vieron obligadas a responder a la situación creada por la gran distribución minorista para mejorar sus resultados. En ambos casos, la estrategia de la industria y de la gran distribución acabó modificando y ampliando la oferta, es decir, lo que el consumidor tenía a su alcance, y el propio consumo.

En esta etapa la marca propia de la distribución se conceptuó, sobre todo, como un producto estándar más barato, que en algunos casos llegó a tener ciertos problemas de calidad. Fueron necesarios bastante tiempo y una estrategia específica de calidad, así como la entrada de enseñas de la distribución de prestigio en ella para cambiar la imagen en el público (Langreo, 2004).

\section{CUADRO 5}

Principales empresas de distribución alimentaria en España, 1985^

\begin{tabular}{lcc}
\hline & Cifra negocio** $^{*}$ & Empleo \\
\hline 1. Hipermercados Pryca, S. A. & 93.395 & 4.995 \\
2. Sociedad Auxiliar de Distribución, S. A. (Continente) & 75.000 & 2.800 \\
3. Alcampo, S. A. & 51.000 & 2.850 \\
4. Simago, S. A. & 44.197 & 4.400 \\
5. Makro, Autoservicio Mayorista, S. A. & 34.404 & 1.139 \\
6. Eroski, Sociedad Cooperativa & 30.155 & 1.379 \\
7. Mercados en Origen, S. A. (Merco) & 28.655 & 964 \\
8. Mercadona, S. A. & 25.800 & 3.100 \\
9. Distribuciones Giménez y Cia., S. A. (Dirsa) & 22.963 & 910 \\
10. Cooperativa Gruma & 18.400 & 265 \\
11. Hipercor, S. A. & 15.982 & 1.100 \\
12. Kanguro Grandes Superficies & 14.157 & 776 \\
13. Distribuciones Reus, S. A. (Dirsa) & 13.491 & 272 \\
14. Distribuidora Internacional de Alimentación, S. A. (Día) & 13.404 & 1.050 \\
15. Peñagrande & 11.677 & 270 \\
\hline
\end{tabular}

^No se incluyen los grandes almacenes El Corte Inglés (302.635 millones) y Galerías Preciados (72.040 millones).

${ }^{\star}$ Ingresos en millones de pesetas corrientes. Facturación total de la empresa.

Fuente: Fomento de la Producción (1987).

De todos modos, aunque en algunos productos de larga duración y en los de importación se contaba con una gestión centralizada del suministro, las plataformas regionales o zonales de compra y, con frecuencia, incluso las tiendas realizaban en buena medida su propia gestión de compras y la dirección estimulaba la competencia entre los distintos estamentos para conseguir precios más baratos. Buena parte del suministro se realizaba desde 
grandes suministradores y los productos frescos, minoría en los hipermercados, se adquirían en los mercados mayoristas en destino, en España en gran parte en los mercas.

Poco antes de la entrada en la CEE (1986), en España convivían grandes empresas de capital francés (Castro, 2010), que lideraban el sector, contaban con hipermercados y luego fueron instalando supermercados, con firmas españolas de distintos tipos e importantes cadenas voluntarias (asociadas) que habían seguido el antiguo modelo (Cuadro 5). Entre las empresas españolas había algunas importantes cooperativas (Eroski, Gruma) y asociaciones de comerciantes junto a firmas regionales de capital familiar local. No hubo ninguna iniciativa, ni desde el sector agrario ni desde el industrial para participar en el sector de la distribución.

El panorama en la CEE continental ofrecía dos modelos empresariales con filosofías diferentes: el modelo francés, que se asentó en España, y el modelo alemán, más centrado en superficies comerciales menores y en cuya estrategia predominaban los precios bajos y la garantía sanitaria por encima de cualquier otra referencia.

En este periodo una de las grandes alarmas que manifestó la Federación de Industrias de la Alimentación y la Bebida (FIAB) fue el problema financiero que causaban a la industria las fórmulas de pago diferidas (mientras la distribución cobraba en el momento), que incluían un riesgo adicional sin los controles necesarios, lo que, ante una quiebra, podría poner en apuros a segmentos muy amplios del sistema alimentario español (Jordana, 1994). Esta forma de pagos fue una de las claves en su financiación y facilitó un crecimiento muy rápido que encajaba con las necesidades de ingresos de los ayuntamientos.

La industria alimentaria, en esta etapa, empezó a perder el papel hegemónico dentro del sistema alimentario y tuvo que responder a las modificaciones de la expansiva gran distribución, que marcó buena parte de su estrategia; pero, también, a importantes cambios sociales ${ }^{8}$. Asimismo, debió adaptarse a la sustitución del sistema español de organización de los mercados agrarios por las Organizaciones Comunes de Mercado (OCM) de la CEE; a la apertura del comercio exterior y el final de sistemas de protección propios en el nuevo contexto institucional que supuso la creación de las comunidades autónomas, por un lado, y la cesión de funciones claves a la Comisión Europea, por otro.

8. Entre ellos, en el contexto del aumento de la renta per cápita, recordemos: la creciente incorporación de la mujer a la vida laboral, la disminución del tamaño de las familias y de los hogares (y su mejor dotación de electrodomésticos), el creciente envejecimiento de la población (Díaz, 2002). 
En estos años, aumentó el flujo de capital internacional, sobre todo europeo, hacia el sistema alimentario español y también hacia su sector industrial ${ }^{9}$. En general, continuó el proceso de reestructuración y concentración de la industria alimentaria ${ }^{10}$, necesaria para responder a la nueva situación ya protagonizada por la fase de la distribución (Rodríguez Zúñiga \& Soria, 1989).

\subsection{De los años noventa a finales de siglo: el Mercado Único y la creciente importancia de los servicios logísticos}

En esta década se produjeron en España cambios muy importantes, tanto en la industria alimentaria como en la gran distribución, que han incidido en la oferta y en el consumo alimentario. La puesta en marcha del Mercado Único en la UE (antes CEE) cambió completamente las relaciones dentro de la Unión al allanarse mucho los inconvenientes al tráfico de mercancías, lo que provocó un aumento de los intercambios de alimentos elaborados y semielaborados y de los mercados de referencia de las empresas (Pinilla \& Serrano, 2009). Asimismo, se afianzó la utilización masiva por la industria y la distribución española de la informatización de procesos y de las comunicaciones y la gestión de clientes, y se abrieron paso enormes mejoras logísticas que colaboraron a cambiar el panorama del sistema alimentario.

\subsubsection{Gran distribución: la centralización de las compras y el auge de la imagen de las enseñas}

Sobre todo, fue la distribución la que aprovechó en mayor medida estas innovaciones. Si ya desde los ochenta el escaneado de los productos con lectores ópticos había facilitado el control de la demanda a los distribuidores, frente a los fabricantes, desde los noventa se centralizó el análisis de la información producida en los distintos puntos de venta en la oficina central de la empresa distribuidora. La rapidez en la reposición de productos de las estanterías de las tiendas de los minoristas hizo cobrar especial importancia a los

9. Entre 1977 y 1987 la cuota de mercado de las empresas participadas mayoritariamente por capital extranjero aumentó del $18 \%$ al $35 \%$. De las 15 mayores empresas alimentarias españolas en 1987, diez eran foráneas (más de la mitad de su capital social) frente a solo tres en 1977.

10. Si en 1977 un grupo aproximado de 200 empresas agroindustriales absorbía una cuota del mercado español próxima al 50\%, en 1987 esa cuota era cubierta por las 100 primeras empresas. Ese año, las 8 mayores empresas suponían solo el $12 \%$ de las ventas totales, aunque el grado de concentración era muy superior a nivel subsectorial. 
servicios logísticos y a la automatización de la gestión de los nuevos almacenes centrales, dotados con equipos de escaneo (Lescent-Giles, 2005).

Así, los años noventa fueron una etapa clave en la configuración de lo que hoy es la gran distribución. En primer lugar, la entrada en vigor del Mercado Único dentro de la CEE, a la vez que propiciaba crecientes flujos comerciales, primaba una mayor concentración empresarial. En esos años las firmas de la gran distribución empezaron a extenderse por más países de la Comunidad Europea, rompiendo los viejos territorios dominados por los modelos alemán o francés; paralelamente, la distribución británica inició su expansión más allá de las islas ${ }^{11}$.

En España, se duplicó la concentración del sector (si en 1991 las ocho primeras empresas minoristas concentraban el 33\% del mercado, en 2001 ya controlaban el 67\%); con todo, una concentración inferior a la de los países europeos de su entorno (Cruz, Rebollo \& Yagüe, 2003).

\section{CUADRO 6}

Evolución de la cuota de los formatos comerciales alimentarios, $1987-2007 \star(\%)$

\begin{tabular}{lrrrr}
\hline & $\mathbf{1 9 8 7}$ & $\mathbf{1 9 9 5}$ & $\mathbf{2 0 0 0}$ & $\mathbf{2 0 0 7}$ \\
\hline Comercio tradicional & 53,0 & 35,6 & 32,2 & 28,0 \\
Supermercados & 30,0 & 35,5 & 41,0 & 45,0 \\
Hipermercados & 3,5 & 16,8 & 19,0 & 17,0 \\
Resto & 13,5 & 12,1 & 7,8 & 10,0 \\
Total & $\mathbf{1 0 0 , 0}$ & $\mathbf{1 0 0 , 0}$ & $\mathbf{1 0 0 , 0}$ & $\mathbf{1 0 0 , 0}$ \\
\hline
\end{tabular}

*Gasto alimentario doméstico.

Fuente: Cruz, Rebollo y Yagüe (2003) y Martín Cerdeño (2008) a partir de datos del Ministerio de Agricultura y Pesca, Alimentación y Medio Ambiente/Ministerio de Medio Ambiente y Medio Rural y Marino.

En esta década de continua pérdida de peso del comercio tradicional alimentario, la creciente competencia entre empresas de la gran distribución minorista obligó a buscar mecanismos que incrementasen las visitas a las tiendas y, con ello, las ventas: se produjo el auge de la fórmula supermercado dentro de las ciudades, que se comportaba como tienda de proximidad, compatible todavía con un pequeño avance del peso de los hipermercados ${ }^{12}$.

11. A principios del siglo XxI, el capital de la gran distribución minorista francesa -difundido especialmente en la Europa del sur- controlaba el 46,1\% del mercado español, frente al 17,4\% alemán (HAYLEY, 2007). A mediados de los años noventa se produjo en España la llegada de los grandes grupos de hard-discount (Lidl).

12. Desde finales de siglo se ha asistido a la saturación del formato de hipermercados (CuESTA, 2004), a la que no ha sido ajena la nueva y restrictiva ley de Comercio (1996). Así, las cadenas de su- 
La entrada con fuerza de la gran distribución minorista en la alimentación fresca, más vinculada a la fórmula del supermercado y potenciadora de las visitas a tienda, estableció un nuevo ámbito de competencia con el menguante comercio tradicional.

\section{CUADRO 7}

Evolución de la cuota del comercio tradicional alimentario, 1992-2007(\%)

\begin{tabular}{lrrr}
\hline & $\mathbf{1 9 9 2}$ & $\mathbf{2 0 0 0}$ & $\mathbf{2 0 0 7}$ \\
\hline Productos frescos & 60 & 50 & 37 \\
Productos secos & 31 & 15 & 9 \\
Total alimentación & & $\mathbf{3 2}$ & $\mathbf{2 8}$ \\
\hline
\end{tabular}

Fuente: véase Cuadro 6.

En esta década, la gran distribución minorista consolidó su control de la cadena alimentaria a través de diversas estrategias. Para ello, las grandes empresas multiplicaron sus formatos/enseñas con el ánimo de llegar a todo tipo de público: establecimientos en formas de hipermercados, supermercados de distintos tamaños, tiendas de conveniencia. Además, ampliaron sus gamas y abrieron el abanico de precios y calidades; algunas de ellas buscaron fórmulas y espacios para una alimentación de calidad diferencial.

Se produjo una revisión de las estrategias de la marca de la distribución, con una opción de calidad a buen precio basada en desarrollos sobre la investigación realizada por la industria. Estas marcas, que solo suponían el 7\% en 1990, alcanzaban en 2000 casi el $19 \%$ del mercado y superaban el 30\% en 2010 (Cruz, 2014).

Se establecieron protocolos de producción, que abarcaba desde la producción agraria y ganadera y los insumos empleados a las técnicas industriales y comerciales o la exigencia de trazabilidad. Estos protocolos permitían transmitir rápidamente las tendencias del consumo a los productores, pero también, a través de ellos, la gran distribución acababa asumiendo decisiones empresariales de las fases anteriores de la cadena de producción. Al tiempo, se inició una estrategia tendente a entrar en el capital de algunas empresas suministradoras o incluso mediante la creación de filiales en los escalones anteriores de la cadena de producción.

La centralización creciente de las decisiones de compra, que aumentaba exponencialmente la capacidad negociadora de la gran distribución frente a las restantes fases del sistema alimentario, obligaba a disponer de grandes suministradores que manejasen ga- 
mas enteras, limitaba el acceso al suministro directo de la gran distribución a pocas empresas y obligaba a las fases anteriores a concentrarse y buscar alianzas para seguir en el mercado (Germán, 2017b). Las principales empresas distribuidoras, ahora varias de ellas españolas (Eroski, Mercadona, El Corte Inglés), integraban las funciones mayoristas en un contexto, además, de fuerte concentración de las centrales de compra que redujo las 21 existentes en 1990 a solo 2 a finales de la década (Cruz, Rebollo \&Yagüe, 2003). Esta estrategia jugó un papel decisivo en la concentración en origen y relegó a los operadores que no alcanzaban las condiciones necesarias a mercados más estrechos o incluso marginales. Este recurso creciente a tratar directamente con grandes operadores concentrados de origen y grandes industriales limitaba el ámbito de actuación de los grandes operadores comerciales.

La entrada directa de la gran distribución en el comercio exterior, primero intra y más tarde extracomunitario, jugó un papel creciente en los intercambios de productos finales y en la llegada a los lineales de productos de terceros países.

En conjunto, esta estrategia, que ha continuado y se ha profundizado ya en el siglo XXI, ha provocado cambios de enorme magnitud y profundidad, afectando sobre todo a la posición de la industria alimentaria. A finales de los años noventa la Comisión Europea se planteó el análisis de la gran distribución no sólo por sus efectos en el consumo o en el sector del comercio minorista, sino por su posición de dominio dentro de la cadena de producción de alimentos.

\subsubsection{La industria alimentaria: los cambios de los años noventa}

Dentro del grupo líder industrial, las empresas de rango europeo fueron centralizando sus direcciones y elaborando estrategias para el continente. La liberalización de los movimientos de capitales con la puesta en marcha del Mercado Único favoreció esta tendencia. La caída del Muro de Berlín en 1989 y con ella la expansión de las empresas a los antiguos países del Este también favoreció, tanto la concentración empresarial como la formulación de acuerdos para penetrar los nuevos mercados con menos riesgo.

La industria alimentaria española aceleró su proceso de modernización e internacionalización, aumentando la inversión extranjera y su apertura al exterior (Rama \& Calatrava, 2002; Serrano et al., 2015) ${ }^{13}$. Se consolidó la entrada de capital extranjero en al-

13. Recordemos que esta aceleración se consolidó especialmente desde mediados de los noventa y posibilitó un nuevo saldo comercial alimentario español positivo (CLAR, SERRANo \& PINILLA, 2015). 
gunas ramas: así, el sector lácteo, concretamente el segmento de la leche líquida, vivió una concentración acusada que fue la principal respuesta a la presión de la gran distribución y fue objeto de entrada de capital, sobre todo francés. Pero dentro del grupo líder de la industria alimentaria, que presentaba muchos casos de verticalización, se mantuvo una importante presencia de empresas españolas ${ }^{14}$ (Langreo, 2006). Con todo, el proceso de concentración de la industria alimentaria ${ }^{15}$ fue inferior al alcanzado por la distribución y, por tanto, su capacidad de negociación con ésta (Cruz, Rebollo \& Yagüe, 2003).

A continuación, vamos a señalar algunos rasgos de diversas ramas alimentarias protagonistas del consumo en esta década (carnes, pescados, productos lácteos, frutas y hortalizas) (Cuadro 3) y su creciente diversificación hacia productos más elaborados.

\section{CUADRO 8}

Evolución en la compra de carne, lácteos y pescados en los hogares, 1990-2000

\begin{tabular}{lrrr}
\hline & $\mathbf{1 9 9 0}$ & $\mathbf{1 9 9 5}$ & $\mathbf{2 0 0 0}$ \\
\hline Total carnes (kg/cap.) & $\mathbf{5 7 , 8}$ & $\mathbf{5 2 , 2}$ & $\mathbf{5 2 , 7}$ \\
Bovino & 7,9 & 7,8 & 7,4 \\
Pollo & 16,1 & 14,3 & 13,8 \\
Ovino y caprino & 3,4 & 3 & 2,8 \\
Cerdo & 8,1 & 7,2 & 10,9 \\
Transformadas & 14,9 & 13,3 & 12,4 \\
\hline & $\mathbf{1 9 9 0}$ & $\mathbf{1 9 9 5}$ & $\mathbf{2 0 0 0}$ \\
\hline Total pescados (kg/cap.) & $\mathbf{2 5 , 2}$ & $\mathbf{2 4 , 6}$ & $\mathbf{2 4 , 3}$ \\
Pescado fresco & 11,8 & 12,8 & 11,7 \\
Pescado congelado & 4,5 & 2,8 & 2,7 \\
Marisco & 6,6 & 6,1 & 6,2 \\
Conservas de pescado & 2,4 & 2,9 & 3,6 \\
\hline & $\mathbf{1 9 9 0}$ & $\mathbf{1 9 9 5}$ & $\mathbf{2 0 0 0}$ \\
\hline Leche líquida (I/cap.) & $\mathbf{1 0 0 , 0}$ & $\mathbf{1 0 8 , 1}$ & $\mathbf{9 9 , 9}$ \\
Esterilizada & 47,7 & 90,9 & 92,6 \\
Pasteurizada & 13,7 & 10,2 & 3,2 \\
Cruda & 21,6 & 9,2 & 4,0 \\
Total derivados lácteos & $\mathbf{1 5 , 6}$ & $\mathbf{2 3 , 3}$ & $\mathbf{2 8 , 6}$ \\
\hline
\end{tabular}

Fuente: Ministerio de Agricultura y Pesca, Alimentación y Medio Ambiente (2005).

14. En 2004, de las 15 principales empresas alimentarias españolas, eran seis las empresas con mayoría de capital extranjero, frente a las 10 de 1987.

15. A finales de siglo las 40 primeras empresas concentraban el $30,2 \%$ del empleo industrial alimentario, cuando a principios de los ochenta suponían el 23,7\% (SERRANo et al., 2015). 
En la industria cárnica aumentó la dimensión media de los mataderos, ya todos frigoríficos, aunque aún estaba muy lejos de la de países como Francia o Alemania. Sin embargo, se mantuvo con buenos resultados una pujante industria pequeña ligada a productos tradicionales (embutidos, salazones) (Langreo, 2007). Frente al estancamiento del consumo per cápita del total de carnes, solo creció la carne de cerdo.

En esta década estaba asentado el consumo de pescado congelado, de cuyo auge eran protagonistas las grandes empresas pesqueras-congeladoras, con o sin marca industrial propia, que venían trabajando desde antes de la transición y que ya habían conseguido implantar el consumo de pescado congelado (algo que nunca se consiguió en las carnes). Estas empresas cambiaron el modo de pescar y toda la cadena de producción, y lanzaron las importantes cadenas de distribución especialistas en congelado. Asimismo, creció el consumo de conservas de pescado.

En el sector lácteo continuaron las tendencias señaladas ya para los años ochenta: el estancamiento del consumo de leche líquida (con creciente protagonismo de la leche esterilizada) contrastó con la prolongación del crecimiento del consumo de los derivados lácteos (Collantes, 2014b, 2015).

En frutas y hortalizas la consolidación de los grandes grupos, situados en Canarias, el sureste peninsular y en Valencia, viene de la mano de los acuerdos con las firmas de la gran distribución europea, en primer lugar de la británica, a través de los cuales se estableció un sistema de producción y control que luego se extendió a las exportaciones a otros países y al mercado interior, donde ha convivido con una oferta muy diversa. Este desarrollo implicó una revolución logística. En cambio, el consumo per cápita de hortalizas frescas en el mercado interior cayó en esta década (de 57,6 a $52 \mathrm{~kg}$ ); también el de frutas frescas (de 99,1 a 84,8 kg) en claro contraste con el crecimiento del consumo de frutas y hortalizas transformadas.

En la producción de pan, la introducción del frío (congelación o refrigeración) cambió el sistema productivo y permitió separar la fabricación propiamente dicha del horneado, dando lugar en esta década a las grandes firmas de masas congeladas. Paralelamente, y como respuesta a la presión sobre los precios de la gran distribución, se produjo un aumento en la gama de productos y en el tipo de establecimientos que los venden, en un contexto de estancamiento del consumo per cápita de pan en esta década finisecular (Langreo, 2001).

En el sector aceitero, en un contexto de caída del consumo per cápita doméstico del total de aceites en esta década (de 18,3 a 14,8 1), algunas cooperativas de segundo grado 
dieron el salto a exportar directamente, especialmente graneles, y empezaron a envasar en grandes cantidades, más allá del suministro al mercado local. Esto, junto a la decisión de la gran distribución de entrar en el mercado de aceite virgen con marca propia, cambió en poco tiempo el equilibrio tradicional en el sector, basado en la elaboración por parte de cooperativas o pequeñas almazaras privadas, y la exportación, refino y envasado en manos de la industria (Langreo, 2010).

En el sector vínico se registró una mejora enorme de la calidad y se estabilizaron las cosechas, fruto de los planes de reestructuración y arranque del viñedo y de la fuerte inversión en las bodegas con apoyo de los fondos comunitarios (Martínez Carrión \& Medina, 2013). Sin embargo, las cooperativas elaboradoras de vino y las pequeñas bodegas no dieron el paso a la exportación directa, debido en buena medida a que la transformación en alcohol, amparada por la OCM, seguía siendo la salida más cómoda. La mejora de la oferta de vino en España, donde siempre ha sido escasa la presencia de productos importados, ha sido fundamental en el cambio de consumo del vino (la caída de su consumo per cápita se ha frenado por el ligero aumento de los vinos de calidad con denominación de origen).

Asimismo, la oferta alimentaria seguía diversificándose dando lugar a los primeros alimentos funcionales, en buena medida también como respuesta a la estrategia de marca blanca de la distribución. Destacaba también el crecimiento de los platos preparados y de los preparados gastronómicos, gamas en las que entraron industrias de diversas ramas analizadas (cárnicas, pescados y conservas vegetales).

En definitiva, en la última década del siglo xx hubo cambios de gran envergadura en la industria y en la distribución alimentaria, que se condicionaron mutuamente y a su vez condicionaron la oferta y el consumo de alimentos. Si el consumo cuantitativo de alimentos ya había tocado techo en los años ochenta, el gasto alimentario continuó desplazándose hacia productos de mayor calidad, más saludables, procesados y personalizados (Cuadro 9): los alimentos que más aumentaron su consumo fueron, además de los ya citados platos preparados, los zumos, refrescos y aguas minerales; pescados en conserva; derivados lácteos y las hortalizas transformadas. En todos ellos, la gran distribución minorista concentraba entre el $73 \%$ y el $94 \%$ de la cuota de mercado frente al escueto peso del comercio tradicional (entre el $4 \%$ y el $13 \%$ ).

Junto al mayor peso de los alimentos-servicio y de los alimentos funcionales, continuó aumentando el consumo de alimentos fuera del hogar (la cuota de éstos últimos ya superaba ampliamente el 40\% en el año 2000). El creciente consumo de alimentos fuera del hogar, junto con el desarrollo del sector de servicios de alimentos (especialmente los 
de comida rápida y los dedicados a la entrega de comida a domicilio) podrían desafiar en el futuro el liderazgo de la gran distribución minorista en la cadena de suministro agroalimentaria (Burch \& Lawrence, 2007) ${ }^{16}$.

\section{CUADRO 9}

Cantidad de alimentos comprados por los hogares, 1990-2001(kg/1 per cáp.)

\begin{tabular}{|c|c|c|c|c|c|c|c|}
\hline & \multirow[t]{2}{*}{1990} & \multicolumn{2}{|c|}{2001 1990-2001 } & \multicolumn{4}{|c|}{ Cuota de mercado por formatos $2001(\%)$} \\
\hline & & \multicolumn{2}{|c|}{ Aumento (\%) } & Tradicional & Supermercado & Hipermercado & Supermercado \\
\hline Platos preparados & 3,4 & 7,2 & 115 & 12,1 & 47,4 & 25,5 & 72,9 \\
\hline Zumos (sin mosto) & 7,6 & 11,6 & 54 & 4,8 & 68,0 & 25,1 & 93,2 \\
\hline Aguas minerales & 20,7 & 39,9 & 92 & 9,5 & 61,0 & 23,9 & 84,8 \\
\hline Refrescos & 23,4 & 39,6 & 69 & 3,9 & 61,8 & 31,9 & 93,7 \\
\hline Pescado en conserva & 2,4 & 3,7 & 52 & 12,8 & 55,0 & 28,3 & 83,3 \\
\hline Derivados lácteos & 15,6 & 28,0 & 79 & 12,1 & 47,4 & 25,5 & 72,9 \\
\hline Frutas/hortalizas transformadas* & 5,8 & 8,3 & 44 & 11,4 & 57,5 & 25,0 & 82,4 \\
\hline
\end{tabular}

*Excepto tomate.

Fuente: Ministerio de Agricultura y Pesca, Alimentación y Medio Ambiente (2005).

\section{CONCLUSIONES}

En este artículo hemos reflexionado sobre la evolución secular de los principales componentes del sistema alimentario español (el sector agrario, la industria y la distribución), sus transformaciones y su influencia en algunos cambios de la dieta de la sociedad española durante los sucesivos periodos del siglo Xx protagonizados por cada uno de dichos componentes.

Hemos mostrado cómo dichas transformaciones están relacionadas con la innovación, con cambios empresariales, con la política agraria y alimentaria, así como con el desarrollo de los mercados, especialmente a partir de la segunda globalización y de la consolidación del Mercado Único europeo.

Así, hemos llevado a cabo un repaso a la creciente y diversificada oferta industrial de alimentos, apoyada en innovaciones tecnológicas que han posibilitado su conservación, inicialmente centrada en la transformación de algunos escasos productos agrícolas y, posteriormente, desde mediados de siglo, en el progresivo protagonismo ganadero-cárnico;

16. En este sentido, estos autores sugieren cómo la creciente participación del capital financiero en el sector podría asimismo suponer en el futuro un cierto desplazamiento del control del sistema alimentario desde la distribución minorista hacia dicho capital. 
y del protagonismo de los productos de primera transformación al mayor peso de productos con sucesivos procesos de transformación, los alimentos-servicio, proceso acelerado en las dos últimas décadas del siglo.

A lo largo de los tres primeros cuartos del siglo se produjeron importantes innovaciones dentro del sector alimentario, tanto en el sector agrario como en el sector industrial, en contraste con una tradicional distribución minorista muy deficiente. Estos avances productivos facilitaron mejoras en la dieta (en cantidad y en diversificación).Ya se habían producido durante el primer tercio del siglo xx; durante el desarrollismo, el renovador impulso del complejo agroindustrial (protagonizado por carne y leche) posibilitó su creciente consumo. La difusión del nuevo modelo comercial de autoservicio se vinculó inicialmente, en especial, al envasado de mercancías secas.

En la cuarta parte del artículo hemos analizado los importantes cambios que se han producido desde el último cuarto del siglo en el ámbito de la distribución alimentaria y su creciente control por parte de las cadenas alimentarias. Estos cambios, conectados a la rápida expansión y la mayor concentración de la gran distribución minorista, han demandado crecientes servicios logísticos y han promovido procesos de integración vertical, así como el avance de las marcas blancas en su oferta alimentaria, aprovechándose de las oportunidades ofrecidas por las nuevas tecnologías de la información y de la comunicación. Unos procesos acelerados en los noventa con la puesta en marcha del Mercado Único y que han condicionado los tradicionales hábitos de compra y de consumo alimentarios -en un contexto de desplazamiento del gasto alimentario hacia la diversificación de productos de mayor calidad, más saludables, procesados y personalizados-; una oferta crecientemente controlada por la gran distribución, compatible con el creciente peso del consumo alimentario fuera del marco doméstico.

\section{AGRADECIMIENTOS}

Una versión inicial de este artículo fue presentada en el XIV Congreso Internacional de Historia Agraria de la SEHA, celebrado en Badajoz del 6-7 de septiembre de 2013, dentro de la sesión «La transición nutricional en perspectiva comparada: mitos y realidades». Este artículo se inserta en el proyecto de investigación 269.187 «Historia de la economía agroalimentaria» del Gobierno de Aragón y la Universidad de Zaragoza, del proyecto ministerial ECO2015-65582-P "La integración de la economía internacional y sus efectos...». Asimismo, se ha beneficiado de las sugerencias emitidas por los evaluadores anónimos de esta revista. 


\section{REFERENCIAS}

ABAD, C. \& NAREDO, J. M. (1997). Sobre la modernización de la agricultura española (1940-1995): De la agricultura tradicional a la capitalización agraria y la dependencia asistencial. En C. Gómez Benito \& J. J. GonzÁlez Rodríguez (Coords.), Agricultura y sociedad en la España contemporánea (pp. 249-316). Madrid: Centro de Investigaciones Sociológicas.

BARCIELA, C. (1985). Intervencionismo y crecimiento agrario en España, 1936-1971. En P. MARTín ACEÑa \& L. PRAdos de LA Escosura (Eds.), La nueva historia económica en España (pp. 285-316). Madrid: Tecnos.

Burch, D. \& Lawrence, G. (Eds.) (2007). Supermarkets and Agri-Food Supply Chains: Transformations in the Production and Consumption of Foods. Cheltenham: Elgar.

CAMilleri, A. (1974). La crisis de la agricultura tradicional. En A. BALlARín et al., La crisis de la agricultura tradicional en España (pp. 47-72). Madrid: Centro de Estudios Sociales del Valle de los Caídos. [Anales de moral social y económica, 34].

CAsares, J. \& Rebollo, A. (1991). Distribución comercial: La aceleración del cambio. 1966-1991:Veinticinco años de revolución comercial en España. Distribución y Consumo, (1), 10-38.

CASTRO, R. (2010). «Máquinas de vender»: Una historia de la gran distribución francesa en España desde los años sesenta. Revista de Historia Industrial, (44), 97-137.

CAstro, R. \& MAIXÉ, J. C. (2015). Structural Change in Peripheral European Markets: Spanish Grocery Retailing, 1950-2007. Fournal of Macromarketing, 35 (4), 448-465.

CATALÁN, J. (1994). Industrialización difusa y desarrollo económico: El retroceso de 19391958. En J. NADAL \& J. CATALÁN (Eds.), La cara oculta de la industrialización española: La modernización de los sectores no líderes (siglos XIX y XX) (pp. 369-396). Madrid: Alianza.

ClaR, E. (2005). Del cereal alimento al cereal pienso: Historia y balance de un intento de autosuficiencia ganadera: 1967-1972. Historia Agraria, (37), 513-544.

CLAR, E. (2008). La soberanía del productor: Industrias del complejo pienso-ganadero e implantación del modelo de consumo fordista en España, 1960-1975. Revista de Historia Industrial, (36), 135-165.

Clar, E., Serrano, R. \& Pinilla, V. (2015). El comercio agroalimentario español en la segunda globalización, 1951-2011. Historia Agraria, (65), 149-186.

Collantes, F. (2014a). La evolución del consumo de productos lácteos en España, 19522007. Revista de Historia Industrial, (55), 103-134.

Collantes, F. (2014b). La distribución minorista de productos lácteos en España (década 1960-presente). Comunicación presentada en el XI Congreso Internacional de la AEHE. Madrid, 4-5 de septiembre. 
Collantes, F. (2015). Patrones de segmentación del consumo de productos lácteos en España, 1958-2006. Investigaciones de Historia Económica, 11 (2), 103-115.

Collantes, F. (2016). A la mesa con Malassis: Modelos de consumo alimentario en la España contemporánea. En D. Gallego, V. Pinilla \& L. G. Germán (Eds.), Estudios sobre el desarrollo económico español (pp. 281-300). Zaragoza: Prensas Universitarias de Zaragoza.

ConTreras, J. (2002). Alimentación y sociedad: Sociología del consumo alimentario en España. En C. Gómez Benito \& J. J. GonzÁlez Rodríguez (Coords.), Agricultura $y$ sociedad en el cambio de siglo (pp. 297-332). Madrid: Mc Graw Hill.

CruZ, I. (2014). Comercio de alimentación: Veinte años de evolución y perspectivas de futuro. Distribución y Consumo, (5), 5-18.

Cruz, I., Rebollo, A. \& Yagüe, M. J. (2003). Concentración y competencia en los canales de distribución de productos alimenticios. Papeles de Economía Española, (96), 112-133.

CuESTA, P. (2004). Treinta años de hipermercados en España: ¿Saturación o renovación? Distribución y Consumo, (74), 46-56.

Cussó, X. \& GARRABOU, R. (2007). La transición nutricional en la España contemporánea: Las variaciones en el consumo de pan, patatas y legumbres (1850-2000). Investigaciones de Historia Económica, (7), 69-100.

DíAz MÉNDEZ, C. (2002). Cambios en el consumo alimentario en España. Abaco, (31), 57-72.

Díaz Méndez, C. \& GonzÁlez Álvarez, M. (2008). Industria y alimentación: De la publicidad referencial a los alimentos funcionales. En C. Díaz MÉndez \& C. Gómez BENITO (Eds.), Alimentación, consumo y salud (pp. 105-129). Barcelona: Fundación «La Caixa».

Di VitTorio, A. \& BARCIELA, C. (Eds.) (2003). Las industrias agroalimentarias en Italia y España durante los siglos XIX y XX. Alicante: Universidad de Alicante.

FERNÁNDEZ, E. (2012). Especialización en baja calidad: España y el mercado internacional del vino, 1950-1990. Historia Agraria, (56), 41-76.

Fomento de LA PROducción (1987). Las 20.000 mayores empresas españolas. Barcelona: Fomento de la Producción.

GaGo, J. M. (2007). El pequeño comercio en la posguerra castellana. Valladolid: Junta de Castilla y León.

Gallego, D. (1993). Pautas regionales de cambio técnico en el sector agrario español (1900-1930). Cuadernos Aragoneses de Economía, 3 (2), 241-276.

GARCÍA, T. (J. Gómez) (1957). La evolución de la cuestión agraria bajo el franquismo. Paris: Parti communiste français.

Garrabou, R. (1997). Políticas agrarias y desarrollo de la agricultura española contemporánea: Unos apuntes. Papeles de Economía Española, (73), 141-148. 
GARRABOU, R. \& Cussó, X. (2009). Dieta mediterránea y transición nutricional moderna en España. En L. Germán, R. Hernández García \& J. Moreno (Coords.), Economía alimentaria en España durante el siglo XX (pp. 25-64). Madrid: Ministerio de Medio Ambiente y Medio Rural y Marino.

GERMÁN, L. (2003). Características del complejo remolachero-azucarero en España, 1882-2000. En A. Di VitToRio \& C. BArCiEla (Eds.), Las industrias agroalimentarias en Italia y España durante los siglos XIX y XX (pp. 335-356). Alicante: Universidad de Alicante.

GERMÁN, L. (2017). Medio siglo de transformaciones en la distribución alimentaria en España (1960-2010): El caso de Aragón. Investigaciones de Historia Económica, 13 (2), 117-126.

Germán, L., Hernández García, R. \& Moreno, J. (Coords.) (2009). Economía alimentaria en España durante el siglo XX. Madrid: Ministerio de Medio Ambiente y Medio Rural y Marino.

Godman, D. \&WATTs, M. (Eds.) (1997). Globalising Food:Agrarian Questions and Global Restructuring. London: Routledge.

GonzÁlez de Molina, M., Soto, D., Aguilera, E. \& Infante, J. (2014). Crecimiento agrario en España y cambios en la oferta alimentaria, 1900-1933. Historia Social, (80), 157-183.

Grandío, A. \& MAIXÉ, J. C. (2008).Vegalsa-Eroski: Homenaje a un emprendedor,Ventura González Prieto y la distribución alimentaria en Galicia, 1945-2007. Vigo: VegalsaEroski.

HAYLEY, N. A. (2007). The Role of Retail Internationalisation in the Establishment of a European Retail Structure. International Fournal of Retail \& Distribution Management, 35 (1), 6-19.

INFANTE, J., Aguilera, E. \& GonZÁleZ DE Molina, M. (2014). La gran transformación del sector agroalimentario español: Un análisis desde la perspectiva energética (19602010). DT-SEHA, (1403).

JoRDANA, J. (1994). La competitividad de la industria alimentaria española. Papeles de Economía Española, (60-61), 264-273.

JUAN I FENOLlaR, R. (1978). La formación de la agroindustria en España (1960-1970): Una aproximación causal y regional. Madrid: Servicio de Publicaciones Agrarias.

Lang, T. \& Heasman, M. (2004). Food Wars: The Global Battle for Mouths, Minds and Markets. London: Earthscan.

Langreo, A. (1978). Análisis de la integración vertical en España. Agricultura y Sociedad, (9), 187-205.

LANGreO, A. (1990). El ganado porcino y las casas de piensos en la ComunidadValenciana: Sus fórmulas de coordinación y su desarrollo histórico. Valencia: Generalitat Valenciana. 
Langreo, A. (1995). Historia de la industria láctea española: Una aplicación a Asturias. Madrid: Ministerio de Agricultura, Pesca y Alimentación.

LANGReo, A. (2001). Harinas, panes, galletas y bollos. Distribución y Consumo, (56), 3553.

LANGReO, A. (2004). ¿Qué está pasando en el sector lácteo? Distribución y Consumo, (77), 93-99.

LANGREO, A. (2006). El grupo de empresas líder del sistema agroalimentario español. Distribución y Consumo, (85), 5-23.

LANGREO, A. (2007). El sistema de producción de carne en España. Estudios Sociales, Revista de Investigación Científica, (31), 40-80.

LANGREO, A. (2008). El sistema alimentario mundial: Principales tendencias y efectos sobre los sistemas alimentarios locales. Distribución y Consumo, (100), 258-276.

LANGREO, A. (2010). La estrategia empresarial en el sector del aceite de oliva y su evolución desde la transición política. Revista de Estudios Empresariales. Segunda época, (1), 7-31.

LANGREO, A. (2011). Gran distribución en Andalucía y su influencia en el sistema alimentario andaluz. Informe anual del sector agrario en Andalucia 2010. Sevilla: Unicaja.

Lescent-Giles, I. (2005). The Rise of Supermarkets in Twentieth-Century Britain and France. En C. SARAsÚA, P. SCHOlliers \& L. van Molle (Eds.), Land, Shops and Kitchens: Technology and the Food Chain in the Twentieth-Century Europe (pp. 188-211). Turnhout: Brepols.

MAIXÉ, J. C. (2009). La modernización de la distribución alimentaria en España, 19471995. Revista de Historia Industrial, (41), 125-160.

MALASsis, L. (1997). Les trois ages de l'alimentaire: Essai sur une histoire social de l'alimentation et de l'agriculture. 2 vols. Paris: Cujas.

MALUQUER DE Motes, J. (2013). La inflación en España: Un índice de precios de consumo, 1830-2012. Estudios de Historia Económica, (64).

Ministerio de Agricultura y Pesca, Alimentación y Medio Ambiente (2005). La alimentación en España, 2004. Madrid: Ministerio de Agricultura y Pesca, Alimentación y Medio Ambiente.

Martín Cerdeño, V. J. (2008). 1987-2007, dos décadas del Panel de Consumo Alimentario: Evolución de los hábitos de compra y consumo en España. Distribución y Consumo, (100), 208-239.

Martínez CARrión, J. M. \& MEdina, F. J. (2013). La competitividad internacional de la industria vinícola española durante la globalización del vino. Revista de Historia Industrial, (52), 139-174.

McMichael, P. (Ed.) (1994). The Global Restructuring of Agro-Food Systems. Ithaca: Cornell University Press. 
NAREDO, J. M. (2004). La evolución de la agricultura en España (1940-2000). Granada: Universidad de Granada.

Nicolau, R. \& Pujol, J. (2011). Aspectos políticos y científicos del modelo de la Transición Nutricional: Evaluación crítica y nuevas perspectivas. En J. BERNABEU \& J. L. BARONA, Nutrición, salud y sociedad: España y Europa en los siglos XIX y XX (pp. 1958). Valencia: Universitat de València.

Nielfa, G. (1989). Las estructuras comerciales en Madrid, 1900-1931: El minifundismo comercial. En L. E. OTERO \& A. BAHAMONDE (Eds.), La sociedad madrileña durante la Restauración, 1876-1931 (pp. 429-458). Vol. I. Madrid: Comunidad de Madrid.

Peinado, M. L. (1985). El consumo y la industria alimentaria en España. Madrid: Instituto de Estudios Agrarios, Pesqueros y Alimentarios.

Pinilla, V. \& AyUdA, M. I. (2009). Foreign Markets, Globalisation and Agricultural Change in Spain. En V. PINILLA (Ed.), Markets and Agricultural Change in Europe from the $13^{\text {th }}$ to the $20^{\text {th }}$ Century (pp. 173-208). Turnhout: Brepols.

Pinilla, V. \& Serrano, R. (2009). Agricultural and Food Trade in the European Union, 1961-2000. En K. K. Patel (Ed.), Fertile Ground for Europe? The History of European Integration and the Common Agricultural Policy since 1945 (pp. 273-300). Baden-Baden: Nomos.

RAMA, R. (1997). Evolución y características de la alimentación fuera del hogar y del consumo de alimentos procesados en España. Agricultura y Sociedad, (84), 107-140.

RAMA, R. \& CALATRAVA, A. (2002). La internacionalización del sistema agroalimentario español: Comercio exterior e inversión extranjera directa. En C. Gómez Benito \& J. J. GonZÁlez Rodríguez (Eds.), Agricultura y sociedad en el cambio de siglo (pp. 181229). Madrid: McGraw-Hill/Interamericana de España.

Reyero, L. (1991). Mercasa: 25 años al servicio de la distribución. Distribución y Consumo, (1), 40-55.

RodRÍGUEZ ZÚÑIGA, M. \& LANGREO, A. (1992). Reestructuración y cambio tecnológico en el complejo cárnico: El sector porcino en España. Revista de Estudios Agrosociales, (159), 67-95.

RODRíGuEZ ZúÑIGA, M., RUIZ-HUERTA, J. \& SORIA, R. (1980). El desarrollo ganadero español: Un modelo dependiente y desequilibrado. Agricultura y Sociedad, (14), 165-183. RODRÍGUEZ ZÚÑIGA, M. \& SORIA, R. (1989). Concentración e internacionalización de la industria agroalimentaria española, 1977-1987. Agricultura y Sociedad, (52), 65-94. SANZ CAÑADA, J. (1997). El sistema agroalimentario español: Cambio estructural, poder de decisión y organización de la cadena alimentaria. En C. Gómez BeNiTo \& J. J. GoNZÁLEZ RodríGUEZ (Eds.), Agricultura y sociedad en la España contemporánea (pp. 355396). Madrid: Centro de Investigaciones Sociológicas.

Sarasúa, C., Scholliers, P. \&VAN Molle, L. (Eds.) (2005). Land, Shops and Kitchens: Technology and the Food Chain in the Twentieth-Century Europe. Turnhout: Brepols. 
SCHRÖTER, H. G. (2008). The Americanisation of Distribution and its Limits: The Case of the German Retail System, 1950-1975. European Rewiew of History, (15), 445-458.

Serrano, R. \& Pinilla, V. (2010). Causes of World Trade Growth in Agricultural and Food Products, 1951-2000: A Demand Function Approach. Applied Economics, 42 (27), 3502-3518.

Serrano, R., García Casarejos, N., Gil Pareja, S., Llorca, R. \& Pinilla, V. (2015). The Internationalisation of the Spanish Food Industry:The Home Market Effect and European Market Integration. Spanish fournal of Agricultural Research, 13 (3), 1-13.

Shaw, G., Louise, C. \& ANDrew, A. (2004). Selling Self-service and the Supermarket: The Americanisation of Food Retailing in Britain, 1945-1960. Business History, 46 (4), 568-582.

SINDE, A. (2014). El abastecimiento de pescado fresco en Barcelona, 1890-1941: Una primera aproximación. XI Congreso Internacional de Historia Económica. Madrid, 45 de septiembre.

Soria, R., Delgado, F. \& Rodríguez ZúÑIGA, M. (1976). El consumo de carnes en España: Estudio, por series cronológicas, de la evolución de la demanda de las carnes de mayor incidencia durante el periodo 1958-1973. Revista de Estudios Agrosociales, (97), 7-37.

SumPSi, J. M. (1997). La modernización de la agricultura y el desarrollo económico. Papeles de Economía Española, (73), 149-159.

Tansey, G. \& Worsley, T. (1995). The Food System. London: Earthscan.

Tió, C. (1982). La política de aceites comestibles en la España del siglo XX. Madrid: Servicio de Publicaciones Agrarias.

ViladomiU, L. (1985). Análisis de la inserción de España en el complejo soja mundial. Agricultura y Sociedad, (34), 151-178. 